\title{
Synthesis and cytotoxicity of natural $(-)$-quinocarcin and its related compounds
}

\author{
Tadashi Katoh and Shiro Terashima* \\ Sagami Chemical Research Center, Nishi-Ohnuma, Sagamihara, Kanagawa 229, \\ Japan.
}

\begin{abstract}
The title synthesis was accomplished by featuring highly diastereoselective reduction of the 1,3-disubstituted isoquinoline 10 to control stereochemistries at the $\mathrm{C}_{5}$ and $\mathrm{C} 11 \mathrm{a}$ positions in $1 \mathrm{a}$ simultaneously in a single step. In vitro cytotoxicity assay of the synthesized quinocarcin congeners including their enantiomers disclosed novel aspects of structure-activity relationships and explored the unnatural 10-decarboxyquinocarcin derivatives $\mathbf{1 b}, \mathbf{3 b}$, and $\mathbf{4 b}$ which are more highly cytotoxic than the corresponding natural 10-carboxy compounds $1 \mathrm{a}, 3 \mathrm{a}$, and $4 a$.
\end{abstract}

$(-)$-Quinocarcin [(-)-1a] isolated from the culture broth of Streptomyces melanovinaceus in 1983, exhibits prominent antitumor activity against various types of mammalian solid tumors (ref.1 and 2). Quinocarcinol (2a), the pharmacologically inactive dihydroderivative of $1 \mathrm{a}$, was also isolated from the same culture broth (ref.1 and 2). While 1a is fairly unstable, cyanation of $1 \mathrm{a}$ with sodium cyanide can produce the more stable hydrogen cyanide adduct, DX-52-1 (3a), which still retains significant antitumor activity (ref. 2). It is also reported that treatment of 3a with silver nitrate cleanly regenerates $1 \mathrm{a}$ (ref. 2 ). The stereostructure of 1 a except its absolute configuration was revealed by a combination of X-ray diffraction and spectral studies to have a novel 8,11-iminoazepino[1,2-b]isoquinoline skeleton with six asymmetric carbons (ref. 2). Although the absolute configuration of la had been suggested based on the computer simulation of binding of 1a to DNA (ref. 3), Garner completed the first asymmetric synthesis of (-)-1a in 1992, leading to confirmation of its absolute configuration (ref. 4). Prior to the Garner's achievement, the total syntheses of ( \pm )-2a and ( \pm )-1a had been accomplished by Danishefsky and Fukuyama, respectively (ref. 5 and 6). As for the mode of antitumor action of 1a, two plausible mechanisms including either DNA alkylation or oxidative DNA cleavage have so far been proposed (ref. 2).

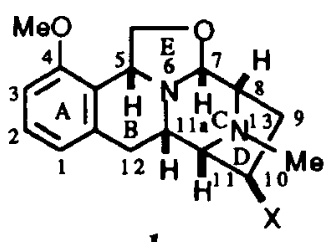

1

a : $\mathrm{X}=\mathrm{CO}_{2} \mathrm{H} ; \mathrm{b}: \mathrm{X}=\mathrm{H}$

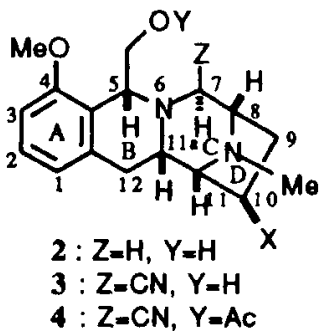

2: $\mathrm{Z}=\mathrm{H}, \mathrm{Y}=\mathrm{H}$

4: $Z=C N, Y=A C$

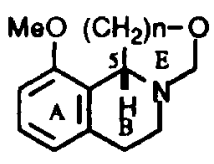

$5: n=1$

$6: n=2$

$7: n=3$

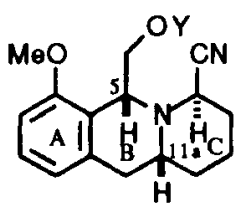

8: $Y=H$

9: $Y=A C$

This report concerns with the total synthesis and in vitro cytotoxicity of natural (-)-1a and its related compounds including their enantiomers. The latter studies disclosed novel aspects of structure-activity relationships for $1 \mathrm{a}$ and let us explore the 10-decarboxy quinocarcin derivatives $\mathbf{1 b}, \mathbf{3 b}$, and $\mathbf{4 b}$ being more highly cytotoxic than the corresponding natural 10-carboxy compounds $1 \mathrm{a}, 3 \mathrm{a}$, and $4 \mathrm{a}$.

\section{Synthesis of $(-)-1 a,(-)-1 b,(+)-3 a,(+)-3 b,(+)-4 a,(+)-4 b$, and Their Enantiomers} (ref. 7 and 8)

At the time when our project was started, the absolute configuration of natural (-)-1a had not been established. Accordingly, prior to embarking on the total synthesis of $(-)-1 a$, the preparations of the ABE ring systems 5-7, the ABC ring systems 8 and 9, and their enantiomers were first carried out to establish an efficient and reliable synthetic method for constructing asymmetric centers at the $\mathrm{C} 5$ and $\mathrm{C} 1 \mathrm{la}$ positions of (-)-1a with the definite absolute configurations (ref. 9 and 10). On the basis of the results accumulated 
Scheme 1

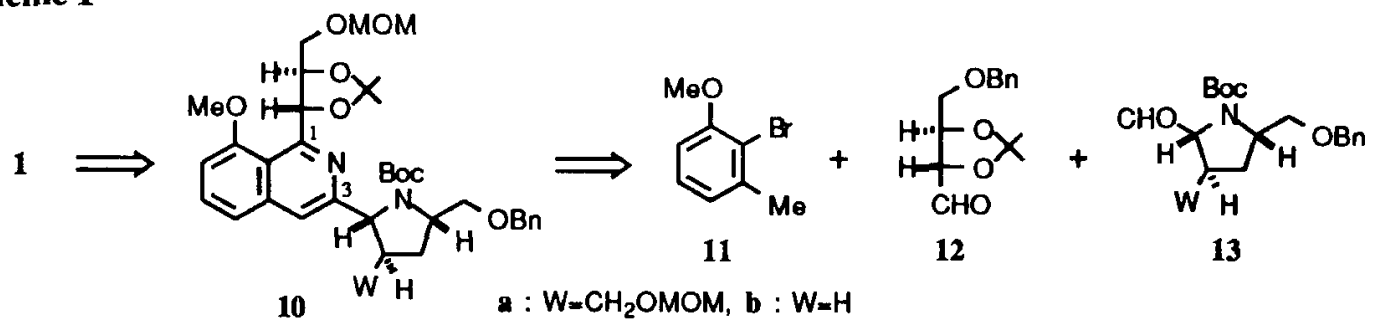

in the successful syntheses of 5-9 and their enantiomers, a novel synthetic strategy for (-)-12 was designed as shown in Scheme 1, which features highly diastereoselective reduction of the 1,3disubstituted isoquinoline 10a accessible from the bromobenzene 11, the D-threose 12, and the 2formylpyrrolidine 13a. In addition to 1a, its 10-decarboxy derivative $1 \mathrm{~b}$ was selected as a target molecule since $1 \mathrm{~b}$ was anticipated to be synthesized more easily than $1 \mathrm{a}$ and antitumor activity of unnatural $1 \mathrm{~b}$ was very intriguing. Preparations of 11 and 12 were readily achieved from commercially available 2-amino-3nitrotoluene and D-tartaric acid, respectively, according to the reported procedures.

As shown in Scheme 2, optically pure 13a,b required for the synthesis of (-)-1a and (-)-1b, were prepared starting with commercially available $(S)$-glutamic acid (14) and/or $(S)$-pyroglutamic acid (17). Thus, $N$-p-methoxybenzylation of $\mathbf{1 4}$ followed by sequential five step operations produced the 2pyrrolidinone 15b. Additional reaction of 15b with Bredereck reagent followed by acidic hydrolysis, reduction, and $O$-protection afforded 15a. Reduction of 15a,b and subsequent aminal formation provided the 2-methoxypyrrolidines $16 \mathrm{a}, \mathrm{b}$. The same compound $16 \mathrm{~b}$ was prepared more effectively from 17 by sequential five step operations. Treatment of $16 \mathrm{a}, \mathrm{b}$ with trimethylsilyl cyanide in the presence of boron trifluoride etherate gave epimeric mixtures of the amino nitriles $18 \mathbf{a}, \mathbf{b}$ and $19 \mathbf{a}, \mathbf{b}$. The desired 2,5-cis isomers 19a,b separated by column chromatography on silica gel were reduced to $13 \mathbf{a}, \mathbf{b}$. On the other hands, the undesired 2,5-trans isomers $18 \mathbf{a}, \mathbf{b}$ were also derived to 13a,b by sequential operations including base catalyzed epimerization of the aldehydes $20 \mathrm{a}, \mathbf{b}$.

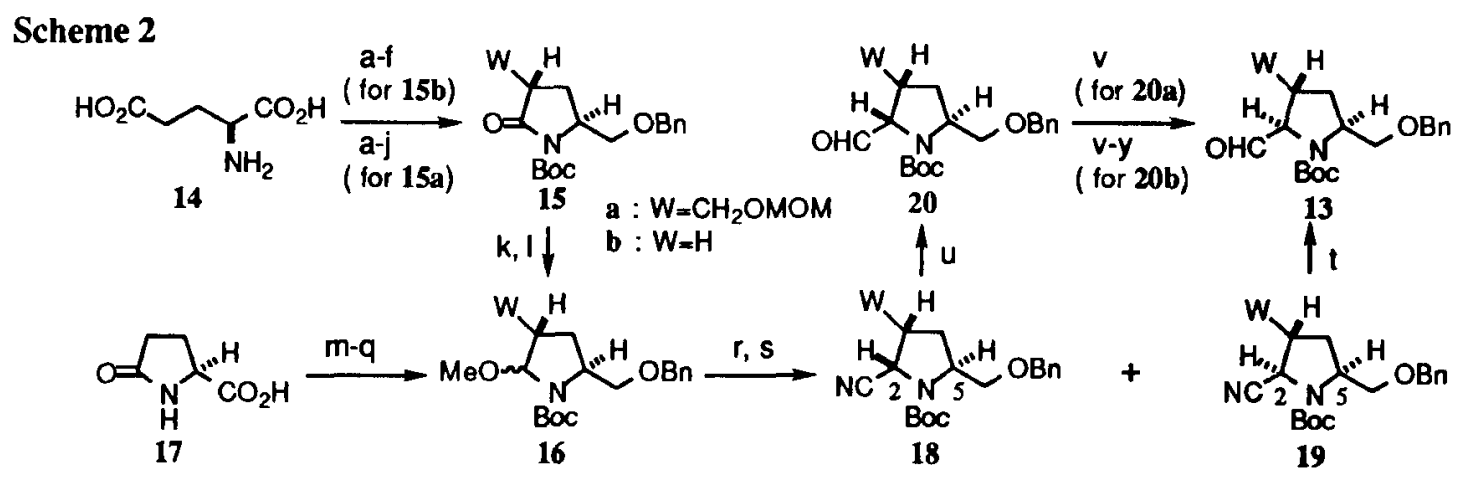

a) p-anisaldehyde, $\mathrm{NaBH}_{4}, 2 \mathrm{MNaOH}, 43 \%$ b) $\mathrm{SOCl}_{2}, \mathrm{EOH}, 88 \%$ c) LiBH, $95 \%$ d) BnBr, $\mathrm{NaH}, 96 \%$ e) CAN, 83\% f) Boc, O, DMAP, EtyN, $98 \%$ g) $\left(\mathrm{Me}_{2} \mathrm{~N}\right)_{2} \mathrm{CHO}^{\prime} \mathrm{Bu}$ h) $1 \mathrm{MHCl}, 88 \%\left(2\right.$ steps) i) $\mathrm{NaBH}_{3} \mathrm{CN}, 81 \%$ j) MOMCl, 'P $\mathrm{r}_{2} \mathrm{EtN}, 82 \%$ k) DIBAL l) PPTS, MeOH, 92\%(tor 15a,b) (2 steps) m) SOCl$, \mathrm{MoOH}, 94 \%$ n) Boc, $\mathrm{O}$, DMAP, El 3 , 100\% o) DIBAL p) PPTS, MoOH, 76\%(2 steps) q) BnBr, NaH, 84\% r) TMSCN, $\mathrm{BF}_{3} \cdot \mathrm{Et}_{2} \mathrm{O}, 95 \%$ (for $18 \mathrm{a}, \mathrm{b}$ and 19a,b)(2 steps)(18a: 19a=69:31, 18b:19b=73:27) s) separation t) DIBAL, 63\%(for 19a), 66\%(for 19b) u) DIBAL, 61\%(for 18a), 63\%(for $18 \mathrm{~b}$ ) v) $\mathrm{K}_{2} \mathrm{CO}_{3}, \mathrm{MeOH}, 100 \%$ w) $\mathrm{NaBH}_{4}, 97 \%$ (cis:trans=78:22) $\mathrm{x}$ ) separation y) (COCl) ${ }_{2}, \mathrm{DMSO}, \mathrm{Cb}_{3} \mathrm{~N}, 97 \%$

With highly functionalized 13a,b in hand, we next focused our attention on the synthesis of $(-)-1 \mathrm{a}$ and (-)-1b. As shown in Scheme 3, lithiation of 11 followed by coupling reaction with 12 gave the ketone 21 having a chiral auxiliary after Collins oxidation of the initially formed secondary alcohol. Exchange of the $O$-protected group provided the methoxymethyl (MOM) ether 23 via the alcohol 22. Deprotonation of 23 followed by the reaction with 13a,b and Jones oxidation produced the diketones $24 \mathbf{a}, \mathbf{b}$, which were further treated with aqueous ammonia to furnish the key intermediates $10 \mathrm{a}, \mathbf{b}$. Crucial reduction of $10 \mathbf{a}, \mathbf{b}$ with sodium cyanoborohydride under acidic conditions proceeded in a highly diastereoselective manner at $0^{\circ} \mathrm{C}$, giving rise to the 1,3-cis-disubstituted tetrahydroisoquinolines $25 \mathrm{a}, \mathbf{b}$ as sole products in excellent yields. The observed high stereoselectivity can be accounted for by the sequential two step asymmetric inductions which proceeded under chelation and stereoelectronic controls, respectively. The reduction products $25 \mathbf{a}, \mathbf{b}$ were elaborated to the aldehydes $28 \mathbf{a}$, b by sequential seven 
Scheme 3
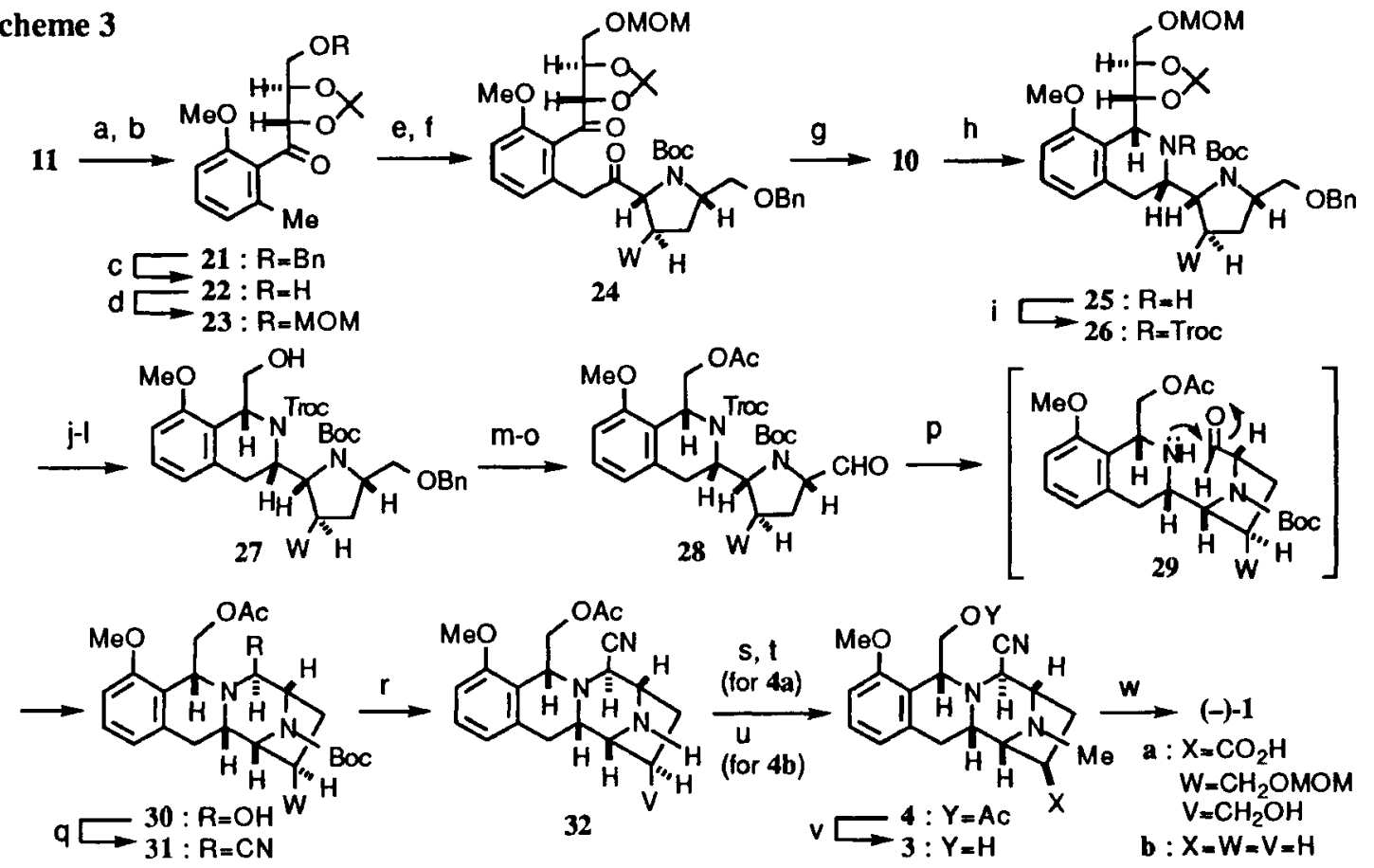

a) "BuLi; 12 b) Collins oxid. 97\%(2 steps) c) $\mathrm{H}_{2}, \mathrm{Pd}(\mathrm{OH})_{2}-\mathrm{C}$ d) MOMCl, $\mathrm{Pr}_{2} \mathrm{EtN}, 90 \%$ (2 steps) e) LDA, TMEDA; 13a or 13b, 51\%(for 13a), $48 \%$ (for $13 \mathrm{~b}$ ) f) Jones oxid., $42 \%$ (for $24 \mathrm{a}$ ), $68 \%$ (for $24 \mathrm{~b}$ ) g) $\mathrm{NH}_{4} \mathrm{OH}, 67 \%$ (for 10a), $72 \%$ (for $10 \mathrm{~b}$ ) h) $\mathrm{NaBH}_{3} \mathrm{CN}, 0.1 \mathrm{M} \mathrm{HCl}-\mathrm{MoOH}, 93 \%$ (for 25a), 98\%(for 25b) i) TrocCl, Py, 85\%(for 26a), 88\%(for 26b) j) $\mathrm{FeCl}_{3}-\mathrm{SiO}_{2}$ (for 26a), 12MHCl(for 26b) k) $\mathrm{NaIO}, \mathrm{l}$ ) $\mathrm{NaBH}, 72 \%$ (for 27a)(3 steps), 68\%(for 27b)(3 steps) m) Ac, O, Py n) $\mathrm{H}_{2}, 10 \% \mathrm{Pd}-\mathrm{C}$ o) (COCl), DMSO, Et $\mathrm{N}, 74 \%$ (for 28a)(3 steps), 82\% (tor 28b) (3 steps) p) Zn, AcOH q) TMSCN, ZnCl, $39 \%$ (for 31a)(2 steps), 56\%(for 31b)(2 steps) r) TFA, 82\%(for 32a), 91\%(for 32b) s) Mel, Pr $\mathrm{EtN} \mathrm{N}$, $68 \%$ t) Jones oxid., $79 \%$ u) $\mathrm{HCHO}, \mathrm{NaBH}_{3} \mathrm{CN}, 72 \%$ v) $1 \mathrm{M} \mathrm{NaOH}, 76 \%$ (for 3a), $83 \%$ for $3 \mathrm{~b}$ ) w) $\mathrm{AgNO}, 81 \%$ [tor (-)-1a], $83 \%[$ for (-). $1 \mathrm{~b}]$

step operations. Removal of the trichloroethoxycarbonyl (Troc) group in $\mathbf{2 8 a}, \mathbf{b}$ afforded the amino aldehydes $29 \mathbf{a}, \mathbf{b}$ which directly cyclized to the unstable hemiaminals $30 \mathbf{a}, \mathbf{b}$. These were immediately derived to the more stable amino nitriles 31a,b. Removal of the tert-butoxycarbonyl (Boc) group in $31 \mathbf{b}$ followed by reductive $N$-methylation yielded the 10 -decarboxylated acetate (+)-4b via amine $32 \mathrm{~b}$. On the other hands, simultaneous removals of both the Boc and MOM groups in 31a followed by $N$-methylation and Jones oxidation gave the 10-carboxylated acetate $(+)-4 a$. Saponification of $(+)-4 a$ and $(+)-4 b$ provided (+)-3a and (+)-3b, which were further treated with silver nitrate to furnish (-)-1a and (-)-1b, respectively. In addition to these products, their enantiomers were similarly prepared starting with $L$ tartaric acid and $(R)$-glutamic acid and/or $(R)$-pyroglutamic acid as starting materials. Ready access of enantiomeric pairs of $\mathbf{1 a}, \mathbf{b}, \mathbf{3 a}, \mathbf{b}$, and $\mathbf{4 a}, \mathbf{b}$ obviously disclosed effectiveness of our explored synthetic scheme.

It was found that the unnatural 10-decarboxyquinocarcin derivatives $(-)-1 \mathbf{b},(+)-3 \mathbf{b}$, and $(+)-4 \mathbf{b}$ are more highly cytotoxic than the corresponding natural 10-carboxy derivatives $(-)-1 a,(+)-3 a$, and $(+)-4 a$ (vide infra). Since a large quantity of $(-)-1 \mathrm{a}$ is available from the fermentation broth of Streptomyces sp., these highly cytotoxic congeners were also prepared from naturally occurring $(-)-1$ a by featuring the Barton's radical decarboxylation (ref. 11). Taking into account the potent cytotoxicity of 10decarboxylated derivatives $(-)-1 b,(+)-3 b$, and $(+)-4 b$, various quinocarcin congeners 33-45 bearing methoxycarbonyl, hydroxymethyl, acetoxymethyl, benzoyloxymethyl, formyl, or fluoromethyl group at their 10 -positions were synthesized starting with $(-)-1$ a by featuring efficient reduction of $(+)-4 a$. From congener 35 , the compound $\mathbf{4 3}$ corresponding to (-)-1a was also prepared (ref. 11).

\section{In Vitro Cytotoxicity of (-)-1a and Its Related Compounds (ref. 11)}

In vitro cytotoxicity assay against $\mathrm{P} 388$ murine leukemia was carried out by employing various structural types of quinocarcin congeners prepared in the course of our synthetic studies. IC50 values $(\mu \mathrm{g} / \mathrm{ml})$ collected are shown in Table 1 . These results clearly disclosed that 10 -decarboxyquinocarcin (1b) and its 7-cyano congeners $3 \mathrm{~b}$ and $4 \mathrm{~b}$ were $10^{1-3}$ times more cytotoxic than the corresponding 10 - 
carboxy compounds $1 \mathrm{a}, 3 \mathrm{a}$, and $\mathbf{4 a}$. It is also noteworthy that $1 \mathrm{a}, 3 \mathrm{a}, \mathbf{4 a}, \mathbf{1 b}, \mathbf{3 b}, \mathbf{4 b}$, and $32 \mathrm{~b}$ bearing natural configurations were found to be $10^{1-4}$ times more cytotoxic than the corresponding enantiomers possessing unnatural absolute configurations. Cytotoxicity of $N_{13}-\mathrm{Boc}$ derivative $31 \mathrm{~b}$ and $\mathrm{N13}-\mathrm{H}$ derivative 32b was obviously inferior to that of the corresponding N13-Me derivative $4 \mathrm{~b}$. The $\mathrm{ABE}$ ring systems 5-7, the $A B C$ ring systems 8 and 9, and their enantiomers showed very weak cytotoxicity. Almost all of the 10-substituted quinocarcin congeners 33-45 derived from 1a exhibit superior cytotoxicity to $1 \mathrm{a}$.
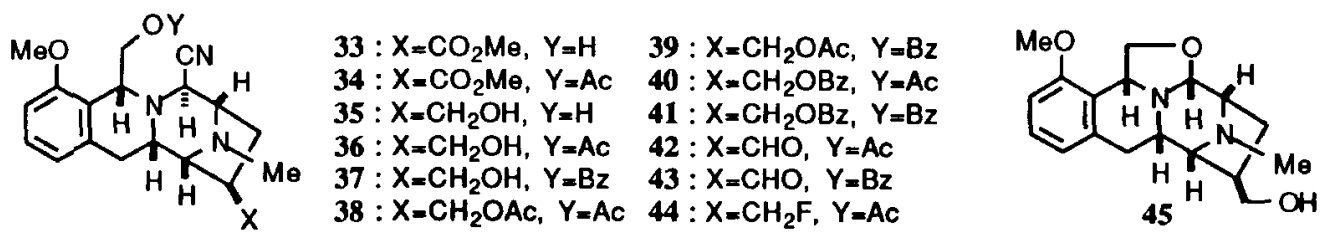

Table 1. In Vitro Cytotoxicity of (-)-Quinocarcin and Its Related Compounds against P388 Murine Leukemia $\left[\mathrm{C}_{50}(\mu \mathrm{gg} / \mathrm{mI})\right]^{\mathrm{a})}$

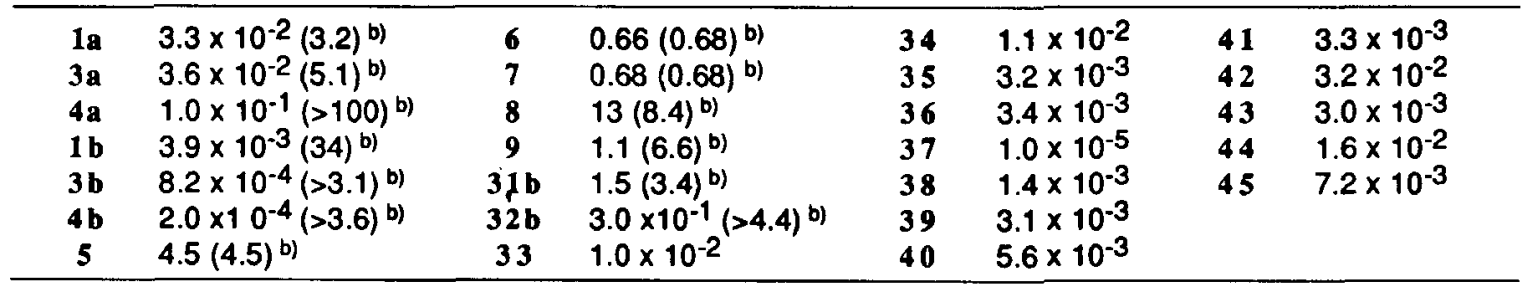

a) Concentration required for $50 \%$ inhibition of the cell growth after incubation for $96 \mathrm{~h}$ at $37^{\circ} \mathrm{C}$ ( initial cell density : $1 \times 10^{4}$ cells $/ \mathrm{ml}$ ). b) Value in parenthesis shows the cytotoxicity of enantiomer.

Summarizing the results of in vitro cytotoxicity assay, it appeared evident that (i) all the carbon framework (the $\mathrm{ABCDE}$ ring system or the $\mathrm{ABCD}$ ring system bearing the $\mathrm{C} 7$-cyano group) with natural absolute configuration is indispensable for significant cytotoxicity, wherein the natural configuration would provide a key structural feature for molecular recognition by DNA, (ii) the N13-methyl group plays an important role for prominent cytotoxicity, and (iii) the C10-carboxyl group is not always necessary for potent activity. The 10-decarboxy congeners $(-)-1 \mathrm{~b},(+)-3 \mathrm{~b},(+)-4 \mathrm{~b}, 37$, and 45 showing promising in vitro cytotoxicity were subjected to in vivo antitumor activity assay, whose detailed results will be reported in due course.

\section{Acknowledgment:}

The authors are grateful to Drs. T. Hirata and H. Saito, Kyowa Hakko Kogyo Co., Ltd., for providing us with a large quantity of $(-)-1 \mathrm{a}$ and $(+)-3 \mathrm{a}$.

\section{References:}

1. K. Takahashi, F. Tomita, J. Antibiot., 36, 468 (1983).

2. T. Katoh, S. Terashima, J. Syn. Org. Chem. Jpn., 52. 556 (1994), and references cited therein.

3. G. C. Hill, T. P. Wuntz, W. A. Remers, J. Comput.-Aided Mol. Des., 2,91 (1988).

4. P. Garner, W. B. Ho, H. Shin, J. Am. Chem. Soc., 114, 2767 (1992). idem, ibid., 115, 10742 (1993).

5. S. J. Danishefsky, P. j. Harison, R. R. Webb II, B. T. O'Neill, J. Am. Chem. Soc., 107, 1421 (1985).

6. T. Fukuyama, J. J. Nunes, J. Am. Chem. Soc., 110, 5196 (1988).

7. T. Katoh, Y. Nagata, Y. Kobayashi, K. Arai, J. Minami, S. Terashima, Tetrahedron, 50, 6221 (1994).

8. T. Katoh, M. Kirihara, Y. Nagata, Y. Kobayashi, K. Arai, J. Minami, S. Terashima, Tetrahedron, 50, 6239 (1994).

9. S. Saito, O. Tamura, Y. Kobayashi, F. Matsuda, T. Katoh, S. Terashima, Tetrahedron, 50, 6193 (1994).

10. S. Saito, K. Tanaka, K. Nakatani, F. Matsuda, T. Katoh, S. Terashima, Tetrahedron, 50, 6209 (1994).

11. T. Katoh, M. Kirihara, T. Yoshino, O. Tamura, F. Ikeuchi, K. Nakatani, F. Matsuda, K. Yamada, K. Gomi, T. Ashizawa, S. Terashima, Tetrahedron, 50, 6259 (1994). 